\title{
School-age outcomes following intraventricular haemorrhage in infants born extremely preterm
}

\author{
Nicky Laura Hollebrandse, ${ }^{1}$ Alicia J Spittle, ${ }^{2,3,4}$ Alice C Burnett, ${ }^{3,5,6,7}$ Peter J Anderson, ${ }^{3,8}$ \\ Gehan Roberts, ${ }^{6,9}$ Lex W Doyle (D) , 3,5,6,10 Jeanie Ling Yoong Cheong 2,3,10
}

\begin{abstract}
- Additional material is
published online only. To view please visit the journal online (http://dx.doi.org/10.1136/ archdischild-2020-318989).
\end{abstract}

For numbered affiliations see end of article.

Correspondence to Professor Jeanie Ling Yoong Cheong, Neonatal Services, Royal Women's Hospital, Parkville, VIC 3052, Australia; jeanie.cheong@thewomens. org.au

Received 10 February 2020 Revised 2 May 2020 Accepted 15 June 2020 Published Online First 30 July 2020

\section{Linked}

- http://dx.doi.org/10.1136/ fetalneonatal-2020-319983

Check for updates

(C) Author(s) (or their employer(s)) 2021. No commercial re-use. See rights and permissions. Published by BMJ.

To cite: Hollebrandse NL, Spittle AJ, Burnett AC, et al. Arch Dis Child Fetal Neonatal Ed 2021;106:F4-F8.

\begin{abstract}
Objective To determine the associations of different grades of intraventricular haemorrhage (IVH), particularly grades 1 and 2, with neurodevelopmental outcomes at 8 years of age in children born extremely preterm.

Design Population-based cohort study.

Setting State of Victoria, Australia.

Patients Survivors born at $<28$ weeks' gestational age $(n=546)$ and matched term-born controls $(n=679)$ from three distinct eras, namely, those born in 1991-1992, 1997 and 2005.
\end{abstract}

Exposure Worst grade of IVH detected on serial neonatal cranial ultrasound.

Outcome measures Intellectual ability, executive function, academic skills, cerebral palsy and motor function at 8 years.

Results There was a trend for increased motor dysfunction with increasing severity of all grades of IVH, from $24 \%$ with no IVH, rising to $92 \%$ with grade $4 \mathrm{IVH}$. Children with grade 1 or 2 IVH were at higher risk of developing cerebral palsy than those without IVH (OR 2.24, 95\% Cl 1.21 to 4.16). Increased rates of impairment in intellectual ability and academic skills were observed with higher grades of IVH, but not for grade 1 and 2 IVH. Parent-rated executive functioning was not related to IVH.

Conclusion While low-grade IVH is generally considered benign, it was associated with higher rates of cerebral palsy in school-aged children born EP, but not with intellectual ability, executive function, academic skills or overall motor function. Higher grades of IVH were associated with higher rates and risks of impairment in motor function, intellectual ability and some academic skills, but not parental ratings of executive function.

\section{INTRODUCTION}

Children born extremely preterm (EP; <28 weeks of gestational age (GA)) are at risk of intraventricular haemorrhage (IVH). In recent years, there has been a decrease in the incidence of IVH; in one large study from $24 \%$ in 1995 to $21 \%$ in $2012 .{ }^{1}$ However, since IVH continues to affect many EP infants it is important to understand its long-term effects. ${ }^{2}$

Grade 3 or 4 IVH reflects more severe pathology and is associated with subsequent neurodevelopmental impairments. ${ }^{34}$ The long-term neurodevelopment of infants with grade 1 or 2 IVH is less clear, with conflicting results reported to date. ${ }^{5-9}$ Moreover, most studies have only associated IVH with early outcomes in the first few years of life, an age when it is not possible to measure outcomes such as executive functioning (EF) or academic skills, which emerge across childhood. ${ }^{10}$

\section{What is already known on this topic?}

- Children born extremely preterm (EP) are at risk for developing intraventricular haemorrhage (IVH) and adverse neurodevelopmental outcomes.

- Grade 3 and 4 IVH is associated with impaired neurodevelopmental follow-up in children born EP.

- The long-term impact of lower grades of IVH is unclear as few studies with school-age followup have been conducted.

\section{What this study adds?}

Lower grades of IVH are associated with higher rates of cerebral palsy at school-age, but not with intellectual, academic or executive functioning.

- Further insight on the association of higher grades of IVH and impaired neurodevelopment at school-age including impaired academic skills.

- Parent-rated executive functioning in daily life is not affected by any degree of IVH.

Glial and neuronal cells arise from the germinal matrix during development. In preterm infants, the germinal matrix undergoes changes after birth involving reduction in size and migration of glial and neuronal cells. Additionally, several studies showed white matter abnormalities on MRI in infants born EP with lower grade IVH. ${ }^{11-13}$ It is therefore hypothesised that even lower grades of IVH might affect the maturation of the brain and thereby result in later neurodevelopmental impairment. ${ }^{48}$

Several studies into school-age have reported minimal influence of IVH grade 1 and 2 on neurodevelopmental outcomes. ${ }^{56}$ However, other studies up to 5.5 years of age have found significant associations of IVH grade 1 and 2 with cerebral palsy (CP) and neurosensory outcomes. ${ }^{78}$ Given the prevalence of low-grade IVH and the uncertainty of its long-term consequences, more research with large sample sizes assessing outcomes beyond early childhood is needed.

The aim of this study was to determine the associations of different grades of IVH with neurodevelopmental outcome at 8 years in EP survivors, particularly of grades 1 and $2 .^{2}$ It was hypothesised that grades 3 and 4 IVH would be related to impaired 
Table 1 Perinatal and sociodemographic characteristics of the EP children assessed at 8 years of age

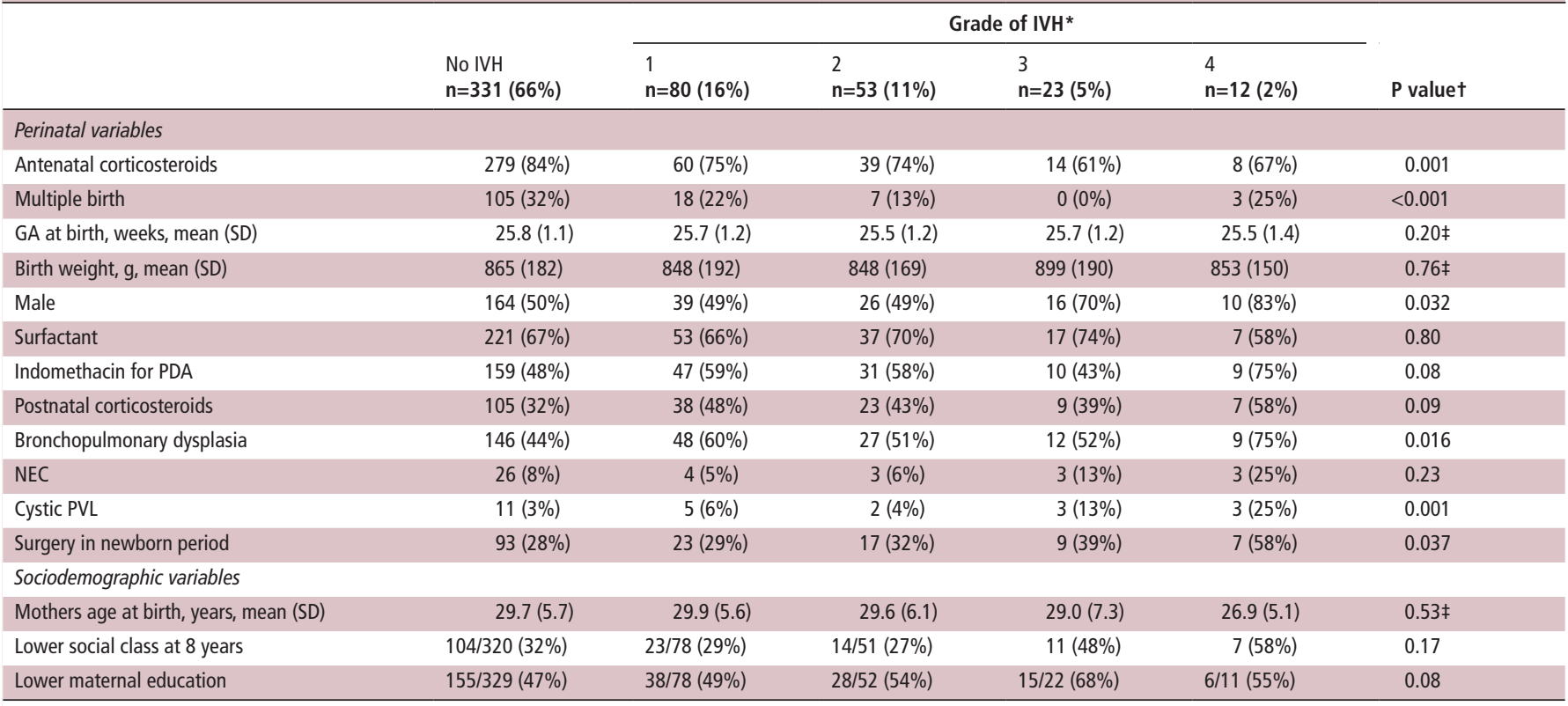

Data are $\mathrm{n}(\%)$, unless otherwise specified.

${ }^{*}$ According to Papile. ${ }^{3}$

†From $\chi^{2}$ trend unless otherwise stated.

¥From one-way ANOVA.

ANOVA, analysis of variance; EP, extremely preterm; GA, gestational age; IVH, intraventricular haemorrhage; NEC, necrotising enterocolitis; PDA, patent ductus arteriosus; PVL, periventricular leukomalacia.

neurodevelopment in all domains, and that grades 1 and $2 \mathrm{IVH}$ would be associated with impaired motor outcomes and milder impairments in intellectual, academic and executive function.

\section{Materials and methods}

Participants comprised three cohorts of children born EP in the state of Victoria in 1991-1992, 1997 and 2005. All survivors were admitted to one of the four tertiary neonatal units in the state. Term-born controls were matched with the EP survivors for the expected date of delivery, child sex, mother's health insurance status (private or public) and country of birth (primarily English speaking or not). Expected due date was based on obstetric ultrasound prior to 20 weeks' gestation and was available for $>90 \%$ of the cohort, otherwise menstrual history was used. Together the EP cohorts comprised 546 survivors over the three eras. ${ }^{14}$

Perinatal data, outlined in table 1 , were collected during the newborn period. Cranial ultrasounds of EP infants were performed at least once during the first week of life, at 28 days and prior to discharge from the tertiary level nursery, as part of standard clinical care. IVH was graded according to Papile, with the worst grade of IVH (1 to 4) on either side recorded. ${ }^{3}$ Additionally, the presence of cystic periventricular leukomalacia (PVL) was recorded.

Children were assessed at 8 years of age, corrected for prematurity to avoid a known bias in cognitive test scores. ${ }^{15}$ Social variables were collected, including lower social class (unskilled occupation of the main income earner in the family, or unemployed) and lower maternal education (<12 years of education for the 1991-1992 and 1997 cohorts and $\leq 12$ years of education for the 2005 cohort). Intellectual, executive, academic and motor outcomes were assessed by psychologists and paediatricians blinded to perinatal details and group status. Cognitive ability was assessed using the Wechsler Intelligence Scale for Children (WISC)-Third Edition (1991-1992 cohort), WISC-Fourth Edition (1997 cohort) and the Differential Ability Scales-Second Edition (2005 cohort). Academic achievement was evaluated using the Wide Range
Achievement Test: version 3 (1991-1992 and 1997 cohorts) or version 4 (2005 cohort). IQ and academic performance z-scores were generated by standardising scores against the mean values of the respective term-born control groups, adjusted for social variables. ${ }^{14}$ Impaired intellectual ability was defined as a z-score $<-2$. Impaired academic skills were defined as any z-score (reading, spelling and/or arithmetic) $<-2$. To assess EF in everyday life, the parent-completed Behaviour Rating Inventory of Executive Functioning was administered, ${ }^{16}$ with impaired EF defined as a standard score $\geq 65$ on the Global Executive Composite, Behavioural Regulation Index or the Metacognition Index.

To assess motor skills, the Movement Assessment Battery for Children (MABC) first edition (1991-1992 and 1997 cohorts) or second edition (2005 cohort) was used. CP was diagnosed in children with loss of motor function along with abnormal tone and tendon reflexes, with severity determined by a functional classification (1991-1992 cohort) or the Gross Motor Function Classification System (1997 and 2005 cohorts). ${ }^{17}$ Motor dysfunction was defined as CP or MABC first edition $<5$ th percentile or MABC second edition $\leq 5$ th percentile.

Data on survival rates, intellectual ability, academic performance, motor function and executive function from these cohorts have been reported previously, but not in relation to grades of IVH. $^{16} 1819$

Data were analysed using IBM SPSS Statistics 22 and STATA/IC 15.1. The associations between different grades of IVH and dichotomous variables, such as rates of impairment at 8 years of age, were assessed using $\chi^{2}$ tests for trend, and between grades of IVH and continuous variables by analysis of variance. Data were then analysed by logistic regression, using generalised estimating equations to account for lack of independence in multiples within the same family. ORs for impairment were computed for IVH grades 1-2 and IVH grades 3-4, compared with the no IVH group as baseline. We added an interaction term between grade of IVH and era to the regression models to assess if any relationships between IVH and 
outcomes differed across eras. We did not consider imputation for missing data at 8 years was necessary because the follow-up rates were high for both cohorts: EP 91.4\% and controls 85\%.

Ethical approval for the different cohorts was obtained through the various Human Research Ethics Committees at the four neonatal units in the state of Victoria, Australia. Written informed consent was obtained from parents of all controls and for children born EP in 2005. In earlier cohorts, the follow-up of children born EP was considered routine clinical care.

\section{RESULTS}

There were 915 EP live births without lethal anomalies over the three eras, $546(59.7 \%)$ of whom survived to the age of 8 years. Of those, 499 (91.4\%) were assessed at the age of 8 years. Children lost to follow-up had similar characteristics to those who were assessed at 8 years except for a higher GA at birth (mean difference 0.38 weeks (95\% CI 0.01 to 0.75$), p=0.03$ ) and lower age of the mother at birth (mean difference -1.94 years $(95 \%$ CI -3.71 to 0.16 ), $\mathrm{p}=0.03$ ) (online supplementary table 1 ). Characteristics of the children were similar across IVH subgroups for GA, birth weight, surfactant treatment and all social variables (table 1 ). There were decreasing trends with worsening grade of IVH for multiple birth and antenatal corticosteroid treatment, and increasing trends for male sex, receiving surgery, postnatal corticosteroids, bronchopulmonary dysplasia and cystic PVL. More than 50\% of all infants were treated with indomethacin for a symptomatic patent ductus arteriosus, but there was little evidence for a trend in indomethacin rates across the IVH subgroups. Ten infants with either grade 3 or 4 IVH developed posthaemorrhagic ventriculomegaly and required surgery for ventricular drainage.

\section{Intellectual ability and EF}

There was weak evidence for more intellectual impairment with increasing severity of IVH (table 2). The increase was most in those with IVH grades 3 and 4 (table 3). Children with IVH showed no increased risk in impaired EF, whether the comparisons were
Table 3 Risks of abnormal outcomes in categories of IVH relative to no IVH

\begin{tabular}{|c|c|c|c|c|}
\hline & \multicolumn{2}{|l|}{ IVH 1 or 2} & \multicolumn{2}{|l|}{ IVH 3 or 4} \\
\hline & OR $(95 \% \mathrm{Cl})$ & $P$ value & OR $(95 \% \mathrm{Cl})$ & $P$ value \\
\hline IQ score $<-2$ SD & $\begin{array}{l}0.78 \\
\text { ( } 0.41 \text { to } 1.48 \text { ) }\end{array}$ & 0.45 & $\begin{array}{l}2.68 \\
\text { (1.21 to } 5.94 \text { ) }\end{array}$ & 0.01 \\
\hline $\mathrm{EF} \mathrm{GEC} \geq 65$ & $\begin{array}{l}1.06 \\
\text { (0.60 to } 1.87 \text { ) }\end{array}$ & 0.84 & $\begin{array}{l}1.17 \\
(0.46 .2 .97)\end{array}$ & 0.75 \\
\hline $\mathrm{EF} \mathrm{BRI} \geq 65$ & $\begin{array}{l}1.12 \\
\text { (0.64 to } 1.95)\end{array}$ & 0.69 & $\begin{array}{l}1.76 \\
\text { (0.75 to } 4.11)\end{array}$ & 0.20 \\
\hline $\mathrm{EF} \mathrm{MI} \geq 65$ & $\begin{array}{l}1.66 \\
\text { (0.98 to } 2.82 \text { ) }\end{array}$ & 0.06 & $\begin{array}{l}1.73 \\
\text { (0.74 to } 4.06)\end{array}$ & 0.21 \\
\hline Any academic skill $<-2$ SD & $\begin{array}{l}0.70 \\
\text { ( } 0.39 \text { to } 1.27 \text { ) }\end{array}$ & 0.24 & $\begin{array}{l}2.91 \\
\text { (1.35 to } 6.27)\end{array}$ & 0.006 \\
\hline Reading $<-2$ SD & $\begin{array}{l}0.85 \\
\text { (0.44 to } 1.66)\end{array}$ & 1.64 & $\begin{array}{l}3.62 \\
\text { (1.59 to } 8.24)\end{array}$ & 0.002 \\
\hline Spelling $<-2$ SD & $\begin{array}{l}0.46 \\
(0.16 \text { to } 1.29)\end{array}$ & 0.14 & $\begin{array}{l}4.48 \\
\text { (1.80 to } 11.2)\end{array}$ & 0.001 \\
\hline Arithmetic $<-2$ SD & $\begin{array}{l}0.61 \\
\text { (0.30 to } 1.27 \text { ) }\end{array}$ & 0.19 & $\begin{array}{l}2.79 \\
\text { (1.20 to } 6.48)\end{array}$ & 0.017 \\
\hline Motor dysfunction & $\begin{array}{l}1.45 \\
\text { (0.93 to } 2.26 \text { ) }\end{array}$ & 0.11 & $\begin{array}{l}4.45 \\
\text { (2.18 to } 9.08)\end{array}$ & $<0.001$ \\
\hline Cerebral palsy & $\begin{array}{l}2.24 \\
\text { (1.21 to } 4.16)\end{array}$ & 0.011 & $\begin{array}{l}8.80 \\
\text { (4.03 to } 19.2 \text { ) }\end{array}$ & $<0.001$ \\
\hline$M-A B C$ & $\begin{array}{l}1.38 \\
\text { (0.87 to } 2.19)\end{array}$ & 0.17 & $\begin{array}{l}4.70 \\
\text { ( } 2.21 \text { to } 9.97 \text { ) }\end{array}$ & $<0.001$ \\
\hline
\end{tabular}

BRI, Behavioural Regulation Index; EF, executive functioning; GEC, Global Executive Composite; IVH, intraventricular haemorrhage; MABC, Movement Assessment Battery for Children.; MI, Metacognition Index.

across all grades of IVH (table 2) or grouped into grades 1-2 and 3-4 (table 3).

\section{Academic skills}

There was strong evidence for increasing trends in impaired academic skills, particularly for reading and spelling, with increasing grade of IVH. Compared with those with no IVH,

Table 2 Impaired cognitive and executive performance related to the grade of IVH

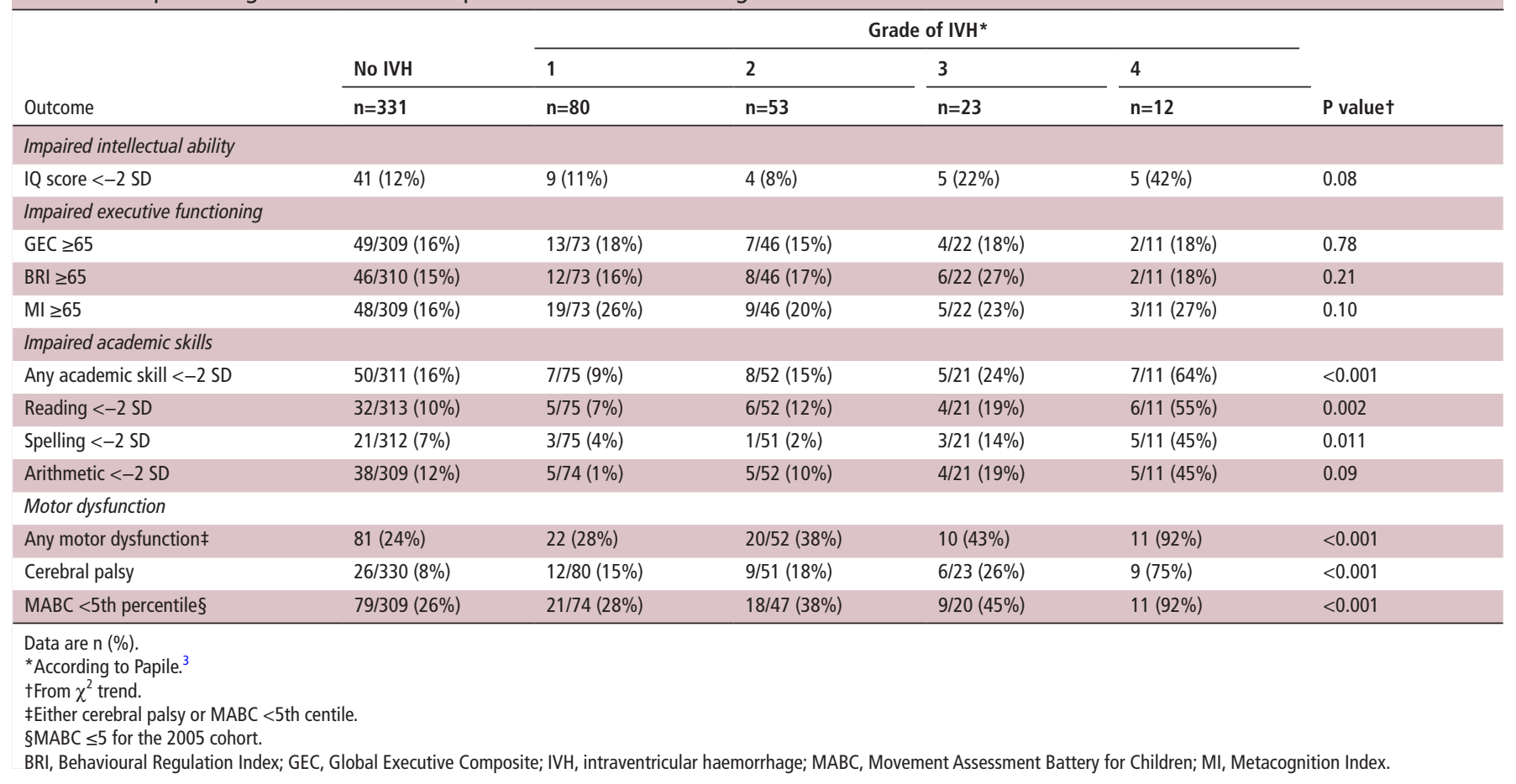


however, only those with grade 3 or 4 IVH were at higher risk of impaired academic scores (table 3).

\section{Motor function}

There was evidence of an increasing trend of motor dysfunction with increasing severity of IVH (table 2). This trend was contributed to by increasing numbers of children with both CP and low scores on the MABC. There was strong evidence for an increased risk of CP in those with grade 1 or 2 IVH compared with no IVH, with little evidence for low motor scores on the MABC alone (table 3). Those with grades 3 or 4 IVH had an increased risk of developing motor impairment compared with no IVH, with strong evidence for both $\mathrm{CP}$ and low scores on the MABC. Of the 62 children with CP, severity was mild in 29 , moderate in 18 and severe in 15. There was no evidence that CP was less severe in children with lower grades of IVH than those with more severe IVH $\left(\chi^{2}\right.$ trend; $\mathrm{p}=0.29$ ). Of the 12 children who had a grade $4 \mathrm{IVH}, 3$ were bilateral. Nine of the 12 children with grade 4 IVH developed CP (table 2). The CP was mild in two cases, moderate in four and severe in three.

There was no evidence that relationships between IVH and outcomes differed across eras (all p-values for interaction terms were $>0.05$ ). Consistent with the findings when outcomes were dichotomised, continuous z-scores for IQ and academic performance deteriorated with increasing severity of IVH, but there was no substantial change in scores for EF across IVH grades (online supplementary table 2).

\section{DISCUSSION}

In our cohort, $27.7 \%$ of the infants who survived to the age 8 years and $33.7 \%$ of those assessed were diagnosed with IVH. Infants with grade 1 or 2 IVH had a higher risk of having a diagnosis of CP at school-age compared with those without IVH. However, grades 1 and 2 IVH were not associated with impaired intellectual, academic or executive function. Higher grade IVH (3 or 4) was associated with poorer intellectual, academic and motor impairment, but not parent-rated EF compared with no IVH.

There are several other studies, with follow-up beyond 2 years of age, that reported absence of significant differences in intellectual outcomes in those with IVH grade 1 or 2 compared with those without IVH. ${ }^{5-7}$ However, results have been inconsistent in studies with a follow-up until 2 years of age. ${ }^{2021}$ Few studies have reported outcomes on academic skills specifically; our current and previous studies show similar findings regarding increased risks of impaired academic outcomes only in those with grade 3 or $4 \mathrm{IVH}^{5}{ }^{5}$

EF includes higher-order cognitive skills supporting goal-directed behaviour and independent problem solving,. ${ }^{22} 23$ The absence of associations between the grade of IVH and parent-rated EF in our sample was surprising. Several studies have reported poor EF in children born EP compared with term-born controls, ${ }^{16}{ }^{24-26}$ but few studies have specifically related EF to the severity of IVH. Vohr et al reported that children at 16 years of age with IVH grade 2 had significantly greater odds of low scores on cognitive flexibility, verbal memory and efficient retrieval of phonological information compared with children without IVH and IVH grade $1 .{ }^{27}$ In the current study, we used parental ratings of behavioural EF rather than direct assessment, which can yield different results. Hence, more extensive research, complementing parental reports with performance-based measurements, is needed. As EF skills continue to develop until early adulthood, assessing EF beyond school-age is essential.

Several other studies have reported associations between IVH grade 1 to 2 and motor impairment. ${ }^{78} 1826$ An Australian study $(n=1472)$ reported higher rates of $\mathrm{CP}$ at 2 to 3 years in those with grade 1 or 2 IVH (10.4\%) compared with those without IVH (6.5\%). ${ }^{8}$ Klebermass-Schrehof et al reported rates of CP at 5.5 years of age in children born $<28$ weeks' gestation with IVH grade $1(34.8 \%)$ and IVH grade $2(55 \%)$ compared with $14.3 \%$ in children with no IVH. ${ }^{7}$ Additionally, the French EPIPAGE study showed a higher prevalence of $\mathrm{CP}$ at 5 years of age in children born $<33$ weeks' GA with IVH grades $1(8 \%)$ and $2(11 \%)$ compared with those with no IVH (4\%). ${ }^{28}$ However, contrary results have also been reported. An American study of 1472 infants born $<27$ weeks' GA at 18 to 22 months did not show any differences of CP rates in those with grades 1 and 2 IVH compared with those without IVH. ${ }^{21}$ We have previously reported a trend in CP from no IVH to IVH grade $3,{ }^{5}$ but the current study in which we added additional cohorts to the first cohort increased the power to find stronger evidence of relationships of lower grade IVH with CP. Grade 3 or $4 \mathrm{IVH}$ was an independent risk factor for developing $\mathrm{CP}$ and motor dysfunction in our study, consistent with recent research. ${ }^{4}$ Strengths of our study include the recruitment of three cohorts over distinct time periods from the same geographic region with contemporaneous term-born matched controls. Outcomes were determined at school-age, which are more meaningful for later life functioning than are outcomes assessed only in the first few years of life. Follow-up rates to school-age were high across the cohorts. Neurodevelopment in several important clinical areas was assessed, and paediatricians and psychologists were blinded to group allocation or previous history to avoid any bias in the assessments.

There are, however, several limitations. Our ultrasound protocols did not include cerebellar or mastoid views across all sites and across all eras, and hence we may have missed some important early pathologies. We did not adjust for later pathology, such as cystic PVL, because it may lie along the causal pathway between IVH and adverse outcomes. Redirection of care might influence the ultimate rates of adverse neurodevelopmental outcomes, but we do not have precise data on how often redirection of care followed a diagnosis of major cranial ultrasound abnormality. Outcome measurements were not identical for the cohorts as different versions were used for the cognitive, academic and motor assessments, but we addressed this problem by computing z-scores relative to contemporaneous controls. To assess executive dysfunction, only parental report was used. However, parents' observations are clinically relevant, since performance within the highly structured neuropsychological testing environment does not perfectly reflect problems faced by children in complex everyday settings. ${ }^{23}$

\section{CONCLUSIONS}

Grades 1 and $2 \mathrm{IVH}$ were associated with a higher risk of CP at 8 years of age. Grades 3 and 4 IVH were associated with poorer intellectual, academic and motor impairment, but not parent-rated EF compared with no IVH. Our study adds to a growing understanding of the negative impact of low-grade IVH on motor development. This emphasises the importance of long-term follow-up and early intervention in children with IVH, as well as parental counselling.

\footnotetext{
Author affiliations

${ }_{1}^{1}$ Faculty of Medical Sciences, Rijksuniversiteit Groningen, Groningen, The Netherlands ${ }^{2}$ Neonatal Services, Royal Women's Hospital, Parkville, Victoria, Australia

${ }^{3}$ Clinical Sciences, Murdoch Children's Research Institute, Parkville, Victoria, Australia ${ }^{4}$ Department of Physiotherapy, University of Melbourne, Parkville, Victoria, Australia

${ }^{5}$ Premature Infant Follow-Up Program, The Royal Women's Hospital, Parkville, Victoria, Australia

${ }^{6}$ Department of Paediatrics, University of Melbourne, Parkville, Victoria, Australia ${ }^{7}$ Neonatal Medicine, Royal Children's Hospital, Parkville, Victoria, Australia
} 
${ }^{8}$ Monash Institute of Cognitive and Clinical Neurosciences, Monash University, Clayton, Victoria, Australia

${ }^{9}$ Centre for Community Child Health, The Royal Children's Hospital Melbourne, Parkville, Victoria, Australia

${ }^{10}$ Department of Obstetrics and Gynaecology, University of Melbourne, Parkville, Victoria, Australia

Acknowledgements The authors acknowledge the following Victorian Infant Collaborative Study Group collaborators: Peter Anderson, Murdoch Children's Research Institute, The Royal Women's Hospital, and University of Melbourne (Melbourne, Australia); Alice Burnett, Murdoch Children's Research Institute, Royal Women's Hospital, University of Melbourne, and The Royal Children's Hospital (Melbourne, Australia); Catherine Callanan, The Royal Women's Hospital; Elizabeth Carse, Monash Medical Centre (Melbourne, Australia); Margaret P Charlton, Monash Medical Centre (Melbourne, Australia); Noni Davis, The Royal Women's Hospital (Melbourne, Australia); Lex W Doyle, The Royal Women's Hospital, Murdoch Children's Research Institute, University of Melbourne, and The Royal Women's Hospital (Melbourne, Australia); Julianne Duff, The Royal Women's Hospital (Melbourne, Australia); Esther Hutchinson, The Royal Women's Hospital and The Royal Children's Hospital (Melbourne, Australia); Marie Hayes, Monash Medical Centre (Melbourne, Australia); Elaine Kelly, The Royal Women's Hospital and Monash Medical Centre (Melbourne, Australia); Katherine J Lee, Murdoch Children's Research Institute (Melbourne, Australia); Marion McDonald, The Royal Women's Hospital (Melbourne, Australia); Gillian Opie, Mercy Hospital for Women (Melbourne, Australia); Gehan Roberts, Murdoch Children's Research Institute, The Royal Women's Hospital, University of Melbourne, and The Royal Children's Hospital (Melbourne, Australia); Andrew Watkins, Mercy Hospital for Women (Melbourne, Australia); Amanda Williamson, Mercy Hospital for Women (Melbourne, Australia) and Heather Woods, Mercy Hospital for Women (Melbourne, Australia). The collaborators were convened by Jeanie Cheong, MD, The Royal Women's Hospital (Melbourne, Australia), Murdoch Children's Research Institute (Melbourne, Australia) and University of Melbourne (Melbourne, Australia). We also acknowledge Professor Arend F Bos, Department of Pediatrics, Division of Neonatology, Beatrix Children's Hospital, University Medical Center Groningen, Groningen, the Netherlands, and the contributions of our participants and their families to this study.

Contributors NLH conceptualised and designed the study, conducted the initial analyses, drafted the initial manuscript, and reviewed and revised the manuscript; AJS conceptualised and designed the study, contributed to data analyses and critically reviewed the manuscript; GR, ACB and PJA contributed to interpretation of data and critical revisions of the manuscript; LWD and JLYC conceptualised and designed the study, designed the data collection and study protocol, coordinated and supervised data collection, contributed to data analyses and critically reviewed the manuscript.

Funding Funded by grants from the National Health and Medical Council (project grant ID 284512; Career Development Fellowship ID 1141354 to JLYC, ID 1108714 to AJS; Senior Research Fellowship ID 1081288 to PJA; Centre of Research Excellence ID 1060733) and the Victorian Government's Operational Infrastructure Support Programme.

\section{Competing interests None declared.}

Patient consent for publication Not required.

Provenance and peer review Not commissioned; externally peer reviewed.

Data availability statement No data are available. Data are not in a repository. It is deidentified participant data, and the data custodian will be me (ORCID: 00000001-5901-0455). No protocol is published, and the statistical analysis plan is in the methods of the paper.

\section{ORCID iD}

Lex W Doyle http://orcid.org/0000-0002-7667-7312

\section{REFERENCES}

1 Yeo KT, Thomas R, Chow SS, et al. Improving incidence trends of severe intraventricular haemorrhages in preterm infants $<32$ weeks gestation: a cohort study. Arch Dis Child Fetal Neonatal Ed 2020;105:145-50.

2 Saigal S, Doyle LW. An overview of mortality and sequelae of preterm birth from infancy to adulthood. Lancet 2008;371:261-9.

3 Papile LA, Burstein J, Burstein R, et al. Incidence and evolution of subependymal and intraventricular hemorrhage: a study of infants with birth weights less than 1,500 GM. J Pediatr 1978:92:529-34.
4 Brouwer AJ, Groenendaal F, Benders MJNL, et al. Early and late complications of germinal matrix-intraventricular haemorrhage in the preterm infant: what is new? Neonatology 2014;106:296-303.

5 Sherlock RL, Anderson PJ, Doyle LW, et al. Neurodevelopmental sequelae of intraventricular haemorrhage at 8 years of age in a regional cohort of ELBW/very preterm infants. Early Hum Dev 2005;81:909-16.

6 Ann Wy P, Rettiganti M, Li J, et al. Impact of intraventricular hemorrhage on cognitive and behavioral outcomes at 18 years of age in low birth weight preterm infants. $J$ Perinatol 2015;35:511-5.

7 Klebermass-Schrehof K, Czaba C, Olischar M, et al. Impact of low-grade intraventricular hemorrhage on long-term neurodevelopmental outcome in preterm infants. Childs Nerv Syst 2012;28:2085-92.

8 Bolisetty S, Dhawan A, Abdel-Latif M, et al. Intraventricular hemorrhage and neurodevelopmental outcomes in extreme preterm infants. Pediatrics 2014;133:55-62.

9 Mukerji A, Shah V, Shah PS. Periventricular/Intraventricular hemorrhage and neurodevelopmental outcomes: a meta-analysis. Pediatrics 2015;136:1132-43.

10 Roberts G, Anderson PJ, Doyle LW, et al. The stability of the diagnosis of developmental disability between ages 2 and 8 in a geographic cohort of very preterm children born in 1997. Arch Dis Child 2010;95:786-90.

11 Vasileiadis GT, Gelman N, Han VKM, et al. Uncomplicated intraventricular hemorrhage is followed by reduced cortical volume at near-term age. Pediatrics 2004:114:e367-72.

12 Jeong HJ, Shim S-Y, Cho HJ, et al. Cerebellar development in preterm infants at Term-Equivalent age is impaired after low-grade intraventricular hemorrhage. J Pediatr 2016;175:86-92

13 Tortora D, Martinetti C, Severino M, et al. The effects of mild germinal matrixintraventricular haemorrhage on the developmental white matter microstructure of preterm neonates: a DTI study. Eur Radiol 2018:28:1157-66.

14 Cheong JLY, Lee KJ, Boland RA, et al. Changes in long-term prognosis with increasing postnatal survival and the occurrence of postnatal morbidities in extremely preterm infants offered intensive care: a prospective observational study. Lancet Child Adolesc Health 2018;2:872-9.

15 Wilson-Ching M, Pascoe L, Doyle LW, et al. Effects of correcting for prematurity on cognitive test scores in childhood. J Paediatr Child Health 2014;50:182-8.

16 Burnett AC, Cheong JLY, Doyle LW. Biological and Social Influences on the Neurodevelopmental Outcomes of Preterm Infants. Clin Perinatol 2018:45:485-500

17 Palisano R, Rosenbaum P, Walter S, et al. Development and reliability of a system to classify gross motor function in children with cerebral palsy. Dev Med Child Neurol 1997;39:214-23

18 Cheong JLY, Anderson PJ, Burnett AC, et al. Changing neurodevelopment at 8 years in children born extremely preterm since the 1990s. Pediatrics 2017;139.

19 Spittle AJ, Cameron K, Doyle LW, et al. Motor impairment trends in extremely preterm children: 1991-2005. Pediatrics 2018;141.

20 Patra K, Wilson-Costello D, Taylor HG, et al. Grades I-II intraventricular hemorrhage in extremely low birth weight infants: effects on neurodevelopment. J Pediatr 2006;149:169-73

21 Payne AH, Hintz SR, Hibbs AM, et al. Neurodevelopmental outcomes of extremely lowgestational-age neonates with low-grade periventricular-intraventricular hemorrhage. JAMA Pediatr 2013:167:451-9.

22 Taylor HG, Clark CAC. Executive function in children born preterm: risk factors and implications for outcome. Semin Perinatol 2016:40:520-9.

23 Anderson P. Assessment and development of executive function (EF) during childhood. Child Neuropsychol 2002;8:71-82.

24 Mulder H, Pitchford NJ, Hagger MS, et al. Development of executive function and attention in preterm children: a systematic review. Dev Neuropsychol 2009;34:393-421

25 Orchinik LJ, Taylor HG, Espy KA, et al. Cognitive outcomes for extremely preterm/ extremely low birth weight children in kindergarten. J Int Neuropsychol Soc 2011:17:1067-79.

26 Baron IS, Kerns KA, Müller U, et al. Executive functions in extremely low birth weight and late-preterm preschoolers: effects on working memory and response inhibition. Child Neuropsychol 2012;18:586-99.

27 Vohr BR, Allan W, Katz KH, et al. Adolescents born prematurely with isolated grade 2 haemorrhage in the early 1990s face increased risks of learning challenges. Acta Paediatr 2014;103:1066-71

28 Beaino G, Khoshnood B, Kaminski M, et al. Predictors of cerebral palsy in very preterm infants: the EPIPAGE prospective population-based cohort study. Dev Med Child Neurol 2010:52:e119-25. 